\title{
Droplet Characterization Based on the Simulated Secondary Rainbows
}

\author{
Wenting Wang*, Jiayi Wang, Yide Zhang \\ College of Science, University of Shanghai for Science and Technology, Shanghai, China \\ Email: *wwt960912@163.com
}

How to cite this paper: Wang, W.T., Wang, J.Y. and Zhang, Y.D. (2021) Droplet Characterization Based on the Simulated Secondary Rainbows. Optics and Photonics Journal, 11, 133-139. https://doi.org/10.4236/opj.2021.116011

Received: May 31, 2021

Accepted: June 8, 2021

Published: June 11, 2021

Copyright $\odot 2021$ by author(s) and Scientific Research Publishing Inc. This work is licensed under the Creative Commons Attribution International License (CC BY 4.0).

http://creativecommons.org/licenses/by/4.0/

(c) (i) Open Access

\begin{abstract}
The droplet size, size distribution, refractive index, and temperature can be measured simultaneously by the rainbow technique. In the present work, the rainbow scattering diagram for a spherical droplet in the secondary rainbow region is simulated by the use of the generalized Lorenz-Mie theory. For achieving high spatial resolution in denser droplet sprays, a focused Gaussian beam is used. For droplet characterization, different inversion algorithms are investigated, which includes trough-trough $\left(\theta_{\min 1}\right.$ and $\left.\theta_{\min 2}\right)$ method and inflection-inflection $\left(\theta_{\text {inf } 1}\right.$ and $\left.\theta_{\text {inf2 }}\right)$ method. For the trough-trough algorithm, the absolute error of the refractive index is between $-6.4 \times 10^{-4}$ and $1.7 \times 10^{-4}$, and the error of the droplet radius is only between $-0.55 \%$ and $1.77 \%$. For the inflection-inflection algorithm, the maximum absolute error of the inverted refractive index is less than $-1.1 \times 10^{-3}$. The error of the droplet radius is between $-0.75 \%$ and $5.67 \%$.
\end{abstract}

\section{Keywords}

Particles Characterization, Rainbow Pattern, Generalized Lorenz-Mie Theory

\section{Introduction}

Liquid atomization and spray play an important role in industrial production, fuel atomization and combustion, spray cooling, and spray drying, etc. [1] [2] [3] [4]. It is of great significance to accurately measure the size, refractive index (temperature), speed, and other parameters of the droplets during the atomization process. Due to inherent non-intrusiveness, the optical technology is used for particle/droplet characterization, including phase Doppler technology, particle image/tracking velocimetry, laser digital holography technology, interferometric particle imaging, time-shift technique, and rainbow technique (or called rainbow refractrometry) [2] [5]-[10]. 
Roth [10] first proposed the rainbow technique method, which is called the standard rainbow technique (SRT). The rainbow pattern was used to measure the refractive index and size of a single spherical droplet. Han [11] measured the small change in the diameter of the liquid column based on the rainbow technique in 1998, assuming that the refractive index is constant. Van Beeck [12] generalized the SRT to global rainbow technology (GRT) to measure the size distribution and temperature of the droplet cloud in the spray. Van Beeck and his colleagues also studied the inversion algorithm of GRT [13] [14]. GRT can be used to measure the mixing ratio of liquid-liquid suspension systems, droplets in large containment vessels, and multiphase flow [14] [15] [16]. In order to have a higher spatial resolution, Gaussian beams are used as a light source. The rainbow pattern of droplets illuminated by Gaussian beams was simulated and used for droplet characterization [17] [18]. It can achieve the purpose of measuring a single tiny droplet under high-density spray conditions. However, only the angles of the first two peaks are used for droplet inversion. This study will discuss the feasibility of other inversion algorithms to measure droplets based on the intensity of scattered light in the secondary rainbow region when Gaussian beams are used as the light source.

The structure of this paper is as follows: In Section 2, inversion schemes for the characterization of droplets are given. In Section 3, based on the secondary rainbow, two inversion schemes are used to compute the refractive index and size of the spherical droplet. Section 4 is devoted to the conclusions.

\section{Inversion Algorithm of Rainbow Technology}

Based on the geometrical optics, the deflection angle $\theta_{p}$ for a ray emerging from a spherical droplet is given as [19] [20]:

$$
\theta_{p}\left(\theta_{i}, m, p\right)=(p-1) \pi+2 \theta_{i}-2 p \arcsin \left(\sin \theta_{i} / m\right), \quad p=0,1,2, \cdots
$$

here $\theta_{i}$ is the incident angle, and $m$ is the relative refractive index of the droplet. The physical meaning of $p[20]$ is $p=0$ is the light ray directly reflected by the spherical droplet, $p=1$ is the light ray directly refracted by the spherical droplet, and $p \geq 2$ is the light ray refracted by the spherical droplet, refracted after $p-1$ times of internal surface reflection. Therefore, the deflection angle of the secondary rainbow $(p=3)$ is:

$$
\theta_{p}=2 \pi+2 \theta_{i}-6 \arcsin \left(\sin \theta_{i} / m\right)
$$

In the above formula, there is an incident angle that minimizes the deflection angle. The minimum deflection angle is called the geometrical optics rainbow angle, and the geometrical optics rainbow angle is given:

$$
\theta_{r g}=2 \pi+2 \sin ^{-1}\left(\frac{9^{2}-m^{2}}{8}\right)-6 \sin ^{-1}\left[\frac{9^{2}-m^{2}}{8 m^{2}}\right]
$$

In Airy theory, the light intensity distribution in the rainbow angle region is expressed by Airy integral. Airy integral $F(z)[20]$ is: 


$$
F(z)=\int_{0}^{\infty} \cos \left[\pi\left(z t-t^{3}\right) / 2\right] \mathrm{d} t
$$

where $z$ is a dimensionless parameter, defined as:

$$
z=(-q)\left[12 /\left(h \pi^{2}\right)\right]^{1 / 2} \alpha^{2 / 3}\left(\theta-\theta_{r g}\right)
$$

where,

$$
\begin{aligned}
& \alpha=2 a \pi / \lambda, \\
& h=\left(p^{2}-1\right)^{2}\left(p^{2}-m^{2}\right)^{1 / 2} /\left[p^{2}\left(m^{2}-1\right)^{3 / 2}\right]
\end{aligned}
$$

Among them, the value of $q$ is -1 or +1 , depending on the angle of the emitted light relative to the incident light [20]. From Airy function, the related $z$ values for the maximum, minimum and inflection point of the curve can be obtained. The normalized light intensity distribution shown in Figure 1 is calculated by using Debye series for spherical water droplet with a radius of $50 \mu \mathrm{m}$ and a relative refractive index of 1.333 . Here, the droplet is illuminated by Gaussian beam, the beam waist radius $\omega_{0}=100 \mu \mathrm{m}$, and the wavelength is $0.6328 \mu \mathrm{m}$. The eigenvalues of the light intensity distribution in the rainbow area (such as $\left.\theta_{\max 1}, \theta_{\max 2}\right)$ have a correspondence with the dimensionless parameter $z$.

Performing algebraic operations on the above formula, the geometrical optics rainbow angle can be obtained from the first two troughs $\left(\theta_{\min 1}\right.$ and $\left.\theta_{\min 2}\right)$ :

$$
\theta_{r g}=\frac{\theta_{\min 1}-C_{1} \theta_{\min 2}}{1-C_{1}}
$$

The refractive index can be obtained from formula (3) and (7), and the droplet radius can be calculated:

$$
a=\frac{2 \lambda}{3 \sqrt{3}}\left[\frac{\left(9-m^{2}\right)^{1 / 2}}{\left(m^{2}-1\right)^{3 / 2}}\right]^{1 / 2}\left(\frac{z_{\min 2}-z_{\min 1}}{\left|\theta_{\min 1}-\theta_{\min 2}\right|}\right)^{3 / 2}
$$

here,

$$
z_{\min 1}=2.4956, z_{\min 2}=4.3632, C_{1}=0.5719
$$

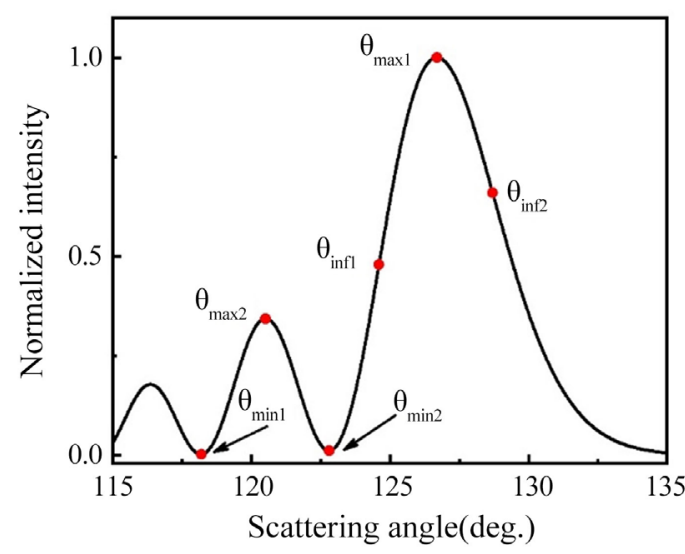

Figure 1. Normalized intensity distribution of the secondary rainbow region. 
For the another algorithm, the angles of the first two inflection points (i.e. $\theta_{\text {infl }}$ and $\left.\theta_{\text {inf2 }}\right)$ of the intensity distribution are employed. The geometrical optics rainbow angle and droplet size are given by:

$$
\theta_{r g}=\frac{\theta_{\text {inf } 1}-C_{2} \theta_{\text {inf } 2}}{1-C_{2}}
$$

and,

$$
a=\frac{2 \lambda}{3 \sqrt{3}}\left[\frac{\left(9-m^{2}\right)^{1 / 2}}{\left(m^{2}-1\right)^{3 / 2}}\right]^{1 / 2}\left(\frac{z_{\text {inf } 2}-z_{\text {inf } 1}}{\left|\theta_{\text {inf } 1}-\theta_{\text {inf } 2}\right|}\right)^{3 / 2}
$$

here,

$$
z_{\text {inf } 1}=0.3276, z_{\text {inf } 2}=1.8724, C_{2}=0.3013
$$

\section{Inversion of Refractive Index and Size of Droplets}

Firstly, according to the Debye series expansion, the scattered light intensity distribution for the secondary rainbow of the spherical droplet is calculated, and the influence of different Gaussian beam waist radius on the peak of the secondary rainbow light intensity distribution is explored. Take the droplet with refractive index of 1.333 and radius of $50 \mu \mathrm{m}$ as an example, as shown in Figure 2. It can be found that when the beam waist radius is $50 \mu \mathrm{m}$, the peak and valley values tend to be stable. Therefore, this study uses Gaussian beam with awaist radius of $50 \mu \mathrm{m}$ as the incident light source and the wavelength of $0.6328 \mu \mathrm{m}$.

The rainbow intensity distribution based on the Debye series expansion simulation only considers the contribution of specific rays. In actual measurement, the total light intensity distribution is produced by the interference of all light rays. These extra interference phenomena cause high-frequency oscillations superimposed on the rainbow pattern, that is, ripple structure. According to the generalized Lorenz-Mie theory (GLMT), the total light intensity distribution in the rainbow region (also known as the rainbow diagram) is calculated. The wavelength of the Gaussian beam is $0.6328 \mu \mathrm{m}$, and the beam waist radius $\omega_{0}=100$ $\mu \mathrm{m}$. The size of the droplet is Between $50 \mu \mathrm{m}$ and $200 \mu \mathrm{m}$. The rainbow diagram of the droplet with a radius of $50 \mu \mathrm{m}$ is shown in Figure 3. The Butterworth digital filter is used for processing to obtain a smooth curve as shown in Figure 1. The cut-off frequency of the low-pass filter is determined based on the elimination of the number of redundant inflection points after roughly estimating the position of the second trough.

The condition of central incidence is studied, where the center of the Gaussian beam is located on the Déscartes ray [18]. According to the inversion algorithm given in Section 2, the calculated refractive index and its absolute error are shown in Figure 4. The calculated radius and its relative error are shown in Figure 5.

For the trough-trough $\left(\theta_{\min 1}\right.$ and $\left.\theta_{\min 2}\right)$ algorithm, the absolute error of the 
refractive index is between $-6.4 \times 10^{-4}$ and $1.7 \times 10^{-4}$ (see Figure $4(\mathrm{~b})$, and the error of the droplet radius is only between $-0.55 \%$ and $1.77 \%$ (see Figure $5(\mathrm{~b})$ ). For inflection-inflection ( $\theta_{\text {inf1 }}$ and $\left.\theta_{\text {inf } 2}\right)$ algorithm, the inverted refractive index is less than $-1.1 \times 10^{-3}$ (see Figure $4(\mathrm{~b})$ ) and error of the droplet radius is between $-0.75 \%$ and $5.67 \%$ (see Figure 5 (b)). Obviously, both algorithms can be used for measuring droplet parameters, but the first algorithm is more suitable for calculating the droplet size than the second algorithm.
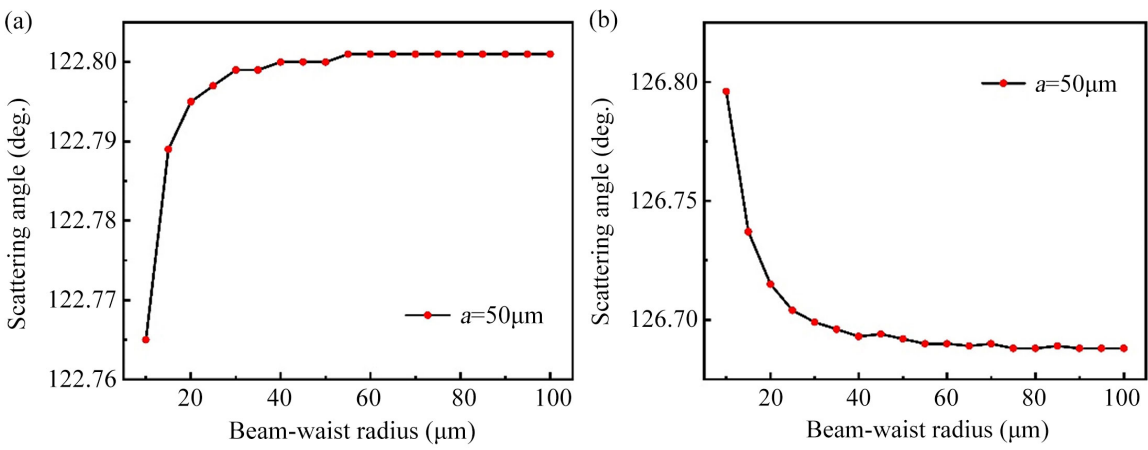

Figure 2. The influence of Gaussian beam incidence with different beam waist radius on the peak position of the secondary rainbow light intensity distribution of the droplet: (a) for the first peak; (b) for the first trough.

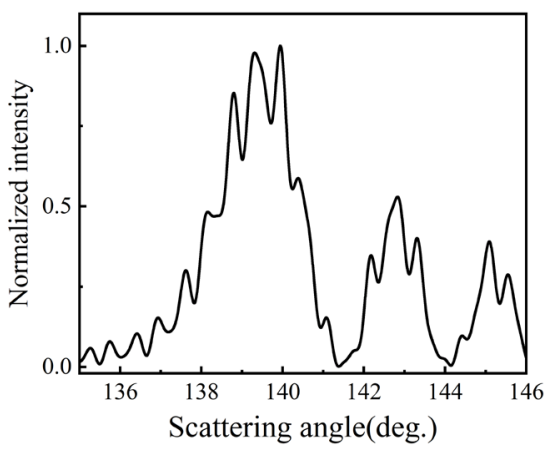

Figure 3. Normalized light intensity distribution for the secondary rainbow of a spherical droplet with $r=50 \mu \mathrm{m}$ illuminated by Gaussian beam.
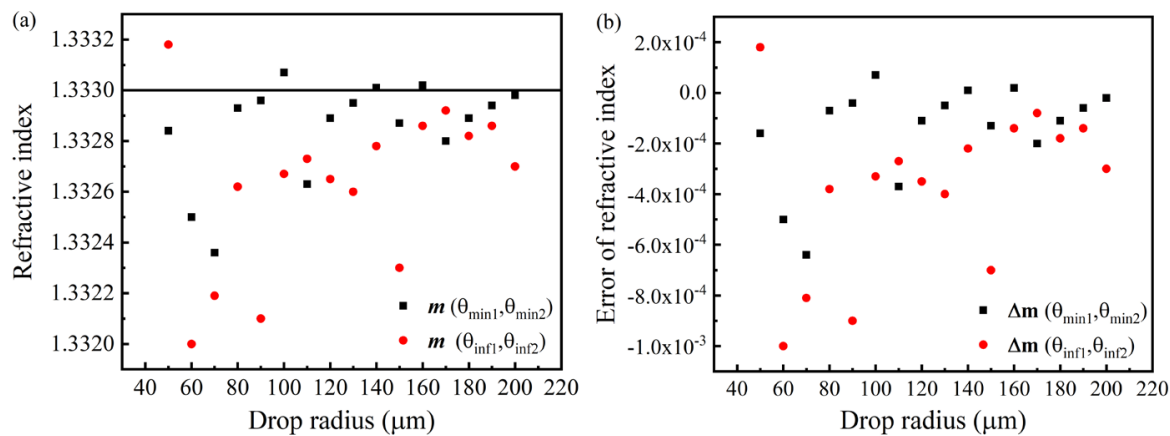

Figure 4. (a) The comparison between the refractive index of the droplet extracted from the secondary rainbow pattern and the true value; (b) The absolute error of the calculated refractive index. 

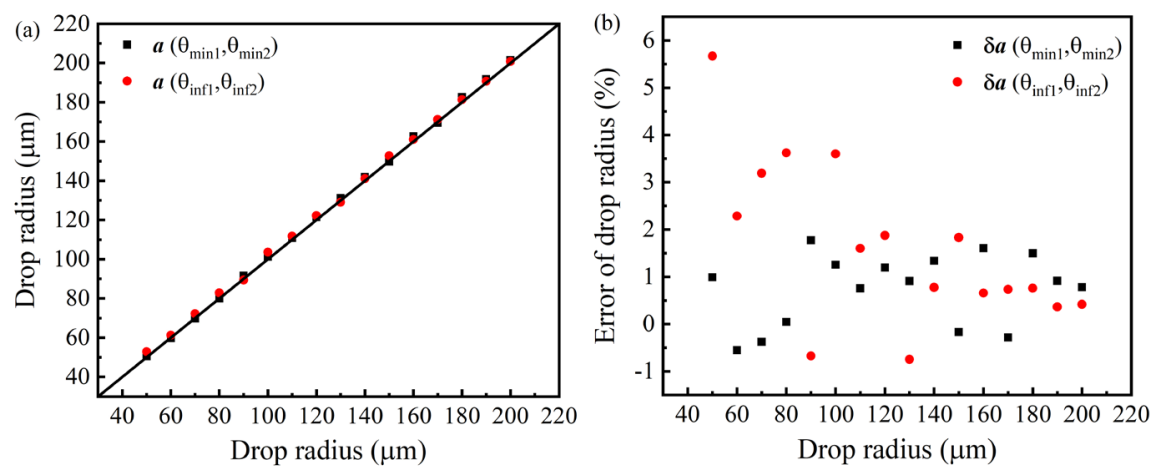

Figure 5. (a) Comparison of the droplet radius extracted from the second-order rainbow pattern with the true value; (b) The relative error of the calculated droplet radius.

\section{Summary}

The generalized Lorenz-Mie theory is used to simulate the rainbow scattering pattern of the droplets in the secondary rainbow region. The low-pass filter passes the simulated rainbow pattern to obtain the characteristic angles. Two different inversion algorithms are studied to invert the droplet parameters. The results show that different inversion algorithms are also feasible to measure droplet information. In particular, for the first algorithm, the error of the droplet radius is even less than $1.77 \%$. However, in this study, the selection of the cut-off frequency of the digital low-pass filter requires artificial evaluation of the angular position of the rainbow diagram. Therefore, we need to develop a better method to select the cutoff frequency or find a better filter, which we need to study further.

\section{Conflicts of Interest}

The authors declare no conflicts of interest regarding the publication of this paper.

\section{References}

[1] Tropea, C. (2011) Optical Particle Characterization in Flows. Annual Review of Fluid Mechanics, 43, 399-426.

https://doi.org/10.1146/annurev-fluid-122109-160721

[2] Sazhin, S. (2014) Droplets and Sprays. Springer, London. https://doi.org/10.1007/978-1-4471-6386-2

[3] Dames, P., Gleich, B., Flemmer, A., Hajeket, K., Seidl, N., Wiekhorst, F., Eberbeck, D., Bittmann, I., Bergemann, C., Weyh, T., Trahms, L., Rosenecker, J. and Rudolphal, C. (2007) Targeted Delivery of Magnetic Aerosol Droplets to the Lung. Nature Nanotechnology, 2, 495-499. https://doi.org/10.1038/nnano.2007.217

[4] Nascimento, L.F., Saldarriaga, C.V., Vanhavere, F., D’Agostino, E., Defraene, G. and Deene, Y.D. (2013) Characterization of OSL $\mathrm{Al}_{2} \mathrm{O}_{3}: \mathrm{C}$ Droplets for Medical Dosimetry. Radiation Measurements, 56, 200-204. https://doi.org/10.1016/j.radmeas.2013.01.048

[5] Albrecht, H.E., Borys, M., Damaschke, N. and Tropea, C. (2003) Laser Doppler and Phase Doppler Measurement Techniques. Springer-Verlag, Heidelberg. https://doi.org/10.1007/978-3-662-05165-8 
[6] Adrian R.J. and Westerweel, J. (2010) Particle Image Velocimetry. Cambridge University Press, Cambridge UK.

[7] Glover, A.R., Skippon, S.M. and Boyle, R.D. (1995) Interferometric Laser Imaging for Droplet Sizing: A Method for Droplet-Size Measurement in Sparse Spray Systems. Applied Optics, 34, 8409-8421. https://doi.org/10.1364/AO.34.008409

[8] Wu, X.C., Lin, X.D., Yao, L.C., Wu, Y.C., Wu, C.Y., Chen, L.H. and Cen, K.F. (2019) Primary Fragmentation Behavior Investigation in Pulverized Coal Combustion with High-Speed Digital Inline Holography. Energy Fuels, 33, 8126-8134. https://doi.org/10.1021/acs.energyfuels.9b01521

[9] Schäfer, W. and Tropea, C. (2014) Time-Shift Technique for Simultaneous Measurement of Size, Velocity, and Relative Refractive Index of Transparent Droplets or Particles in a flow. Applied Optics, 53, 588-597.

https://doi.org/10.1364/AO.53.000588

[10] van Beeck, J.P.A.J. and Riethmuller, M.L. (1995) Nonintrusive Measurements of Temperature and Size of Single Falling Raindrops. Applied Optics, 34, 1633-1639. https://doi.org/10.1364/AO.34.001633

[11] Han, X.E., Ren, K.F., Wu, Z.S., Corbin, F., Gouesbet, G. and Gréhan, G. (1998) Characterization of Initial Disturbances in a Liquid Jet by Rainbow Sizing. Applied Optics, 37, 8498-8503. https://doi.org/10.1364/AO.37.008498

[12] van Beeck, J.P.A.J., Giannoulis, D. and Zimmer, L. (1999) Global Rainbow Thermometry for Droplet-Temperature Measurement. Optics Letters, 24, 1696-1698. https://doi.org/10.1364/OL.24.001696

[13] van Beeck, J.P.A.J., Zimmer, L. and Riethmuller, M.L. (2001) Global Rainbow Thermometry for Mean Temperature and Size Measurement of Spray Droplets. Particle \& Particle Systems Characterization, 18, 196-204. https://doi.org/10.1002/1521-4117(200112)18:4<196::AID-PPSC196>3.0.CO;2-H

[14] Vetrano, M.R., van Beeck, J.P.A.J. and Riethmuller, M.L. (2004) Global Rainbow Thermometry: Improvements in the Data Inversion Algorithm and Validation Technique in Liquid-Liquid Suspension. Applied Optics, 43, 3600-3607. https://doi.org/10.1364/AO.43.003600

[15] Lemaitre, P., Porcheron, E., Gréhan, G. and Bouilloux, L. (2006) Development of a Global Rainbow Refractometry Technique to Measure the Temperature of Spray Droplets in a Large Containment Vessel. Measurement Science and Technology, 17, 1299-306. https://doi.org/10.1088/0957-0233/17/6/002

[16] Wu, Y.C., Li, C., Cao, J.Z., Wu, X.C., Saengkaew, S., Chen, L.H., Gréhan, G. and Cen, K.F. (2018) Mixing Ratio Measurement in Multiple Sprays with Global Rainbow Refractometry. Experimental Thermal and Fluid Science, 98, 309-316. https://doi.org/10.1016/j.expthermflusci.2018.06.004

[17] Yu, H.T., Sun, H. and Shen, J.Q. (2018) Measurements of Refractive Index and Size of a Spherical Drop from Gaussian Beam Scattering in the Primary Rainbow Region. Journal of Quantitative Spectroscopy and Radiative Transfer, 207, 83-88. https://doi.org/10.1016/j.jqsrt.2017.12.028

[18] Cao, Y.Y., Wang, W.T., Yu, H.T., Shen, J.Q. and Tropea, C. (2020) Characterization of Refractive Index and Size of a Spherical Drop by Using Gaussian Beam Scattering in the Secondary Rainbow Region. Journal of Quantitative Spectroscopy and Radiative Transfer, 242, Article ID: 106785. https://doi.org/10.1016/j.jqsrt.2019.106785

[19] Hulst, H.C.V.D. (1957) Light Scattering by Small Particles. Physics Today, 10, 28-30. https://doi.org/10.1063/1.3060205

[20] Wang, R.T. and Hulst, H.C.V.D. (1991) Rainbows: Mie Computations and the Airy Approximation. Applied Optics, 30, 106-117. https://doi.org/10.1364/AO.30.000106 